\title{
HD Light Functions in Headlights for More Traffic Safety
}

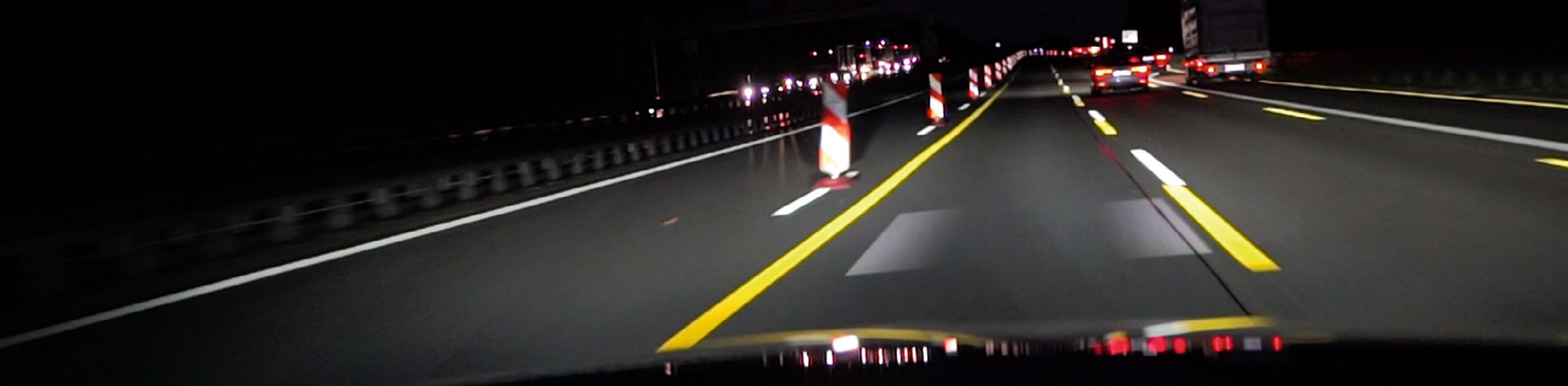

AUTHORS

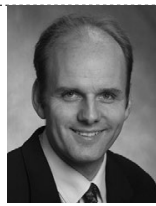

Dr.-Ing. Ernst-Olaf Rosenhahn is Director of the Headlamp Innovations Department at Marelli Automotive Lighting in Reutlingen (Germany).

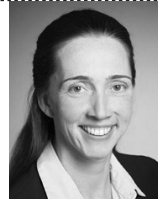

Dr. Ulrike Schlöder is Employee in the Headlamp Innovations Department at Marelli Automotive Lighting in Reutlingen (Germany).

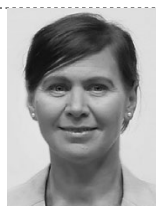

Ilona Rutkiewicz, M. A.

is Employee in the Marketing Department at Marelli Automotive Lighting in Reutlingen (Germany).

Compared to the DMD technology in headlights, the more cost-convenient micro-LED technology can make it possible to spread HD lighting functions to all vehicle segments. The microLED technology saves approximately $30 \mathrm{~W}$ of power consumption, $30 \%$ installation space and $30 \%$ weight per headlamp pair in average. Marelli Automotive Lighting compares both technologies and presents light projections on the ground for assistance functions and warning symbols in more detail.

In motor vehicle headlights, highresolution projection systems based on the so-called HD lighting technologies such as the Digital Mirror Device (DMD) technology are offered as optional equipment in the upper passenger car segment. With their help, light projections onto the roadway or the ground are made technically possible: symbols or assistance functions (warning signs or guiding edges) support the driver during the night-time driving $[1,2]$. These projections must not however be too noticeable so that they do not disturb or distract other road users [3].

In various test series, Marelli Automotive Lighting was able to show that the digital assistance functions have a positive influence on the driving behavior and thus increase traffic safety for other road users as well. In the coming years, further novel HD light functions will increasingly find their way onto the market. In particular, the next generation of high-resolution projection systems based on micro-LED technology will ensure the widespread use of HD light functions in all vehicle segments and will thus become interesting for all vehicle manufacturers. Less complex in structure, the new technology delivers simpler projections in good quality with a lower optical resolution 
system is the micro mirror system with 1.3 million mirrors, each with a size of approximately $8 \times 8 \mu \mathrm{m}$. They are illuminated by an optical system with three high-current LEDs and the reflected luminous flux is projected onto the roadway via projection lens system.

These DMD modules are contrasted by micro-LED light sources as the next generation of high-resolution light, referred to as h-Digi microLED at Marelli Automotive Lighting, FIGURE 1. These semiconductor-based LED arrays can be arranged in a matrix of for example 80 rows and 250 columns. Each LED having an edge length of only 40 to $50 \mu \mathrm{m}$ can be addressed individually by the electronics. This means that around 20,000 pixels in matrix form are available for the high-resolution light distribution. A CMOS layer below the LED matrix ensures that each pixel can be dimmed. As a result, the light distribution patterns and symbols are flexibly generated and displayed in front of the vehicle by projection optics. Here, too, the highresolution illumination field is limited to the central area of the light distribution.

As with DMD technology, a further projection module is used as a partner module to display the basic illumination for low and high beam also with the micro-LED technology. Yet, the microLED system has several advantages over the DMD system: With a slightly larger illumination field, it consumes approximately $30 \mathrm{~W}$ less electrical power consumption on average per vehicle. Thanks to the simplified structure of the optical system, the h-Digi microLED is signifi- cantly smaller than the h-Digi one, which reduces the installation space requirement in the headlight by almost $30 \%$ and a weight saving of approximately $1 \mathrm{~kg}$ per vehicle is achieved.

However, only 20,000 pixels are available for the light distribution with the micro LED system, which reduces the resolution of the projected images. While the DMD system, with its $0.01^{\circ}$ resolution, comes close to the resolution of the human eye, the resolution of the micro-LED system is only $0.1^{\circ}$. Assistance projections on the road are possible, but their size and level of detail must be adapted to the reduced resolution during development. Both technologies require an active cooling by a fan in order to ensure suitable thermal management even at long operating times and high ambient temperatures.

\section{EFFECTIVENESS OF GUIDING PROJECTIONS}

As part of an investigation with two times five subjects, a test vehicle with high-resolution DMD headlights was used. In construction sites, they projected guiding edges onto the road ahead of the car at a distance of 10 to $20 \mathrm{~m}$ [4]. These projections, which show the driver the width of his or her own vehicle, including exterior mirrors, are intended to serve as an assistance function and support the driver in keeping the vehicle in the center of the own lane, TITLE FIGURE.

The lateral position of the vehicle in its lane while driving through construction Lighting since 2018 - support the driver both with assistance projections on the lane and with flexible adaptation of the cut-off line of the low beam and the glare-free high beam to different traffic situations. While the partial high beam can work much more precisely thanks to the high resolution, the range of the full high beam is significantly increased by the additional light intensity of the high-resolution DMD module h-Digi. The module's illumination field is in the central area of the headlight distribution, as this is where the high resolution can generate the greatest benefit for the driver. Automatic control, primarily through signals from a camera located in the vehicle that continuously analyzes the area in front of the passenger car, optimizes safety and comfort for the driver. The heart of the DMD projection
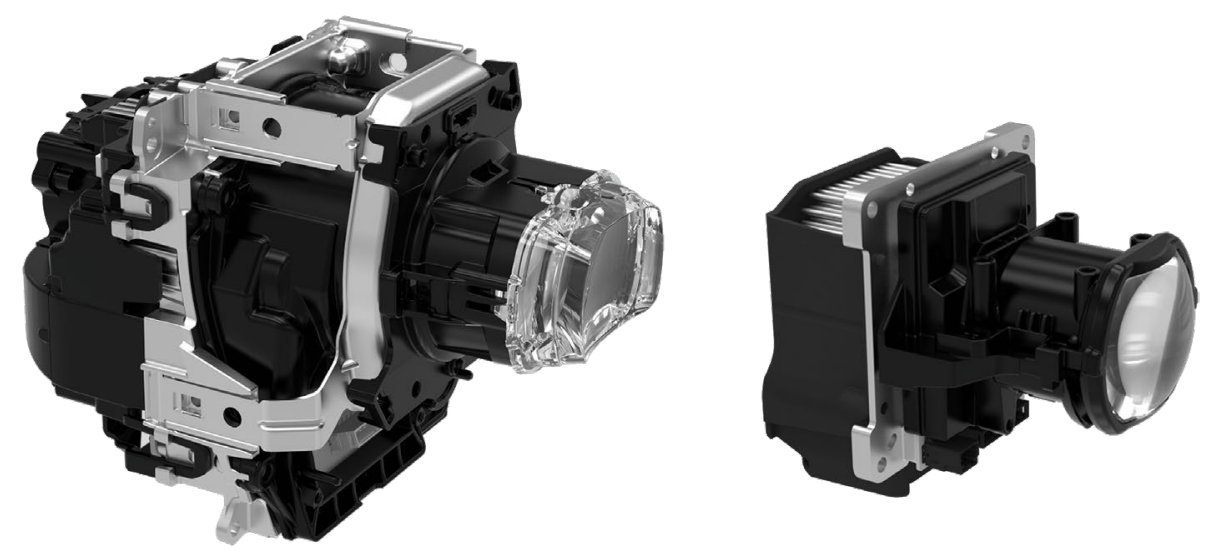

FIGURE 1 On the way to miniaturization of high-resolution lighting systems: the DMD module h-Digi (left) and the micro-LED module h-Digi microLED (right) in comparison (C Marelli Automotive Lighting) 


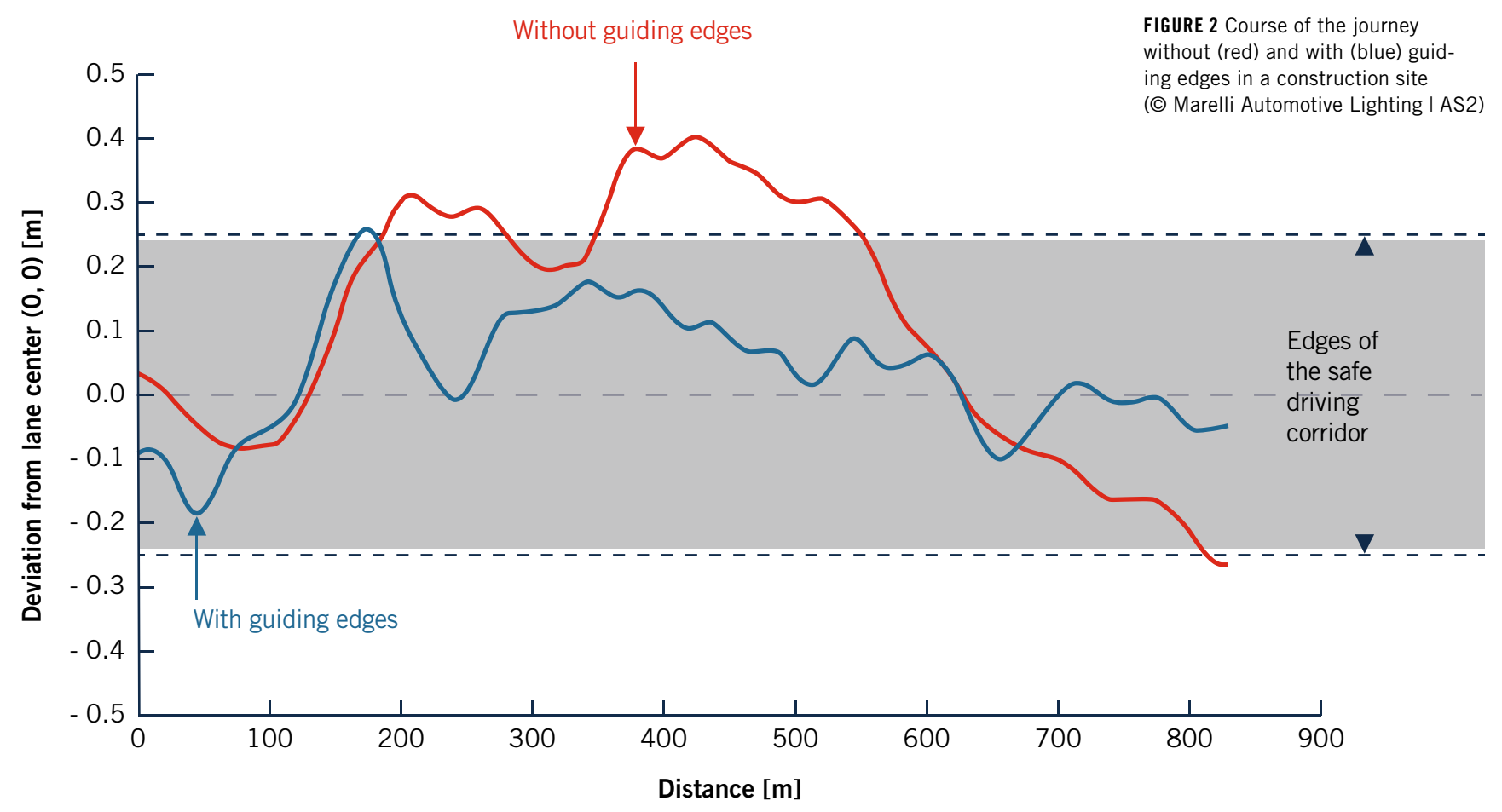

sites was therefore recorded as a measured variable with the support of a camera. To evaluate the effectiveness of the assistance function rated in this test, journeys with and without front projections were compared for each test subject. The investigations were carried out in four different construction site areas on the German A 81 highway (Stuttgart-Heilbronn) over a total distance of $20 \mathrm{~km}$.

An area, the so-called Safe Driving Corridor (SDC) was defined within the driver's lane to assess road safety. For the driving situation examined with a vehicle width of $1.90 \mathrm{~m}$ and a lane width of $2.40 \mathrm{~m}$, the SDC is 1.90 $\pm 0.25 \mathrm{~m}$, an area in which the tires do not leave the specified lane. For a vehicle driving within this corridor, the risk of a side collision with other road users is low. In a comparative analysis, the proportional driving time in the SDC was evaluated.

As an example, FIGURE 2 shows the measured values of a subject driving through one of the construction sites for $800 \mathrm{~m}$. Without the guiding edges, a maximum deviation of $42 \mathrm{~cm}$ to the left and $28 \mathrm{~cm}$ to the right of the lane center was observed. With the guiding edges, these values decreased for the same subject to $28 \mathrm{~cm}$ to the left and $18 \mathrm{~cm}$ to the right.

Without assistance functions, the vehicle of one of the test groups was in the construction site area approximately $38 \%$ of the time outside the SDC. With projected guiding elements, this proportion decreased to $10 \%$. The other group of more experienced drivers was out of the corridor $9 \%$ of the time while no guiding projection was available. Thanks to the usage of the projection edges, they could reduce this percentage to only $1 \%$. It was thus possible to show a significant reduction in the proportion of time outside the safe driving corridor for all drivers, which proves the effectiveness of these projections with regards to road safety.

\section{SIGNALING GROUND PROJECTIONS AROUND THE VEHICLE}

On the way to partially and fully automated driving, there will be continuous
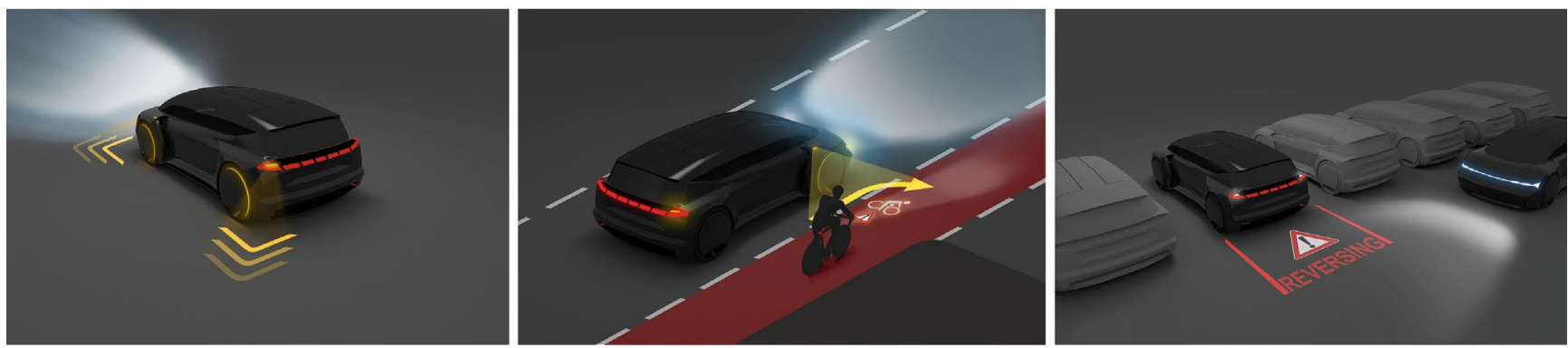

FIGURE 3 Examples of more safety through ground projections - indication of turn direction (left), turning process (center) and leaving the parking lot (right) (C Riccardo Anzaldi I Marelli Automotive Lighting) 
D's SIMULIA

\section{MBS TECH DAYS 2021 MULTIBODY SYSTEM SIMULATION AT ITS BEST}

November 16-18, 2021 | Virtual Event

Join us on November 16-18, 2021 for our MBS Tech Days. For the first time, this event is globally accessible online.

During this three-day live event, we will focus on user multibody simulation experiences and the latest developments in Simpack.

Our agenda features a lineup of presenters like Daimler Truck, Mercedes Benz, Honda, Stellantis, Hyundai, DB Systemtechnik, MesH Engineering, Virtual Research Vehicle, Goldwind, China Academy of Railway Sciences, RWTH Aachen, TU Berlin, Universität der Bundeswehr and much more.

\section{Highlights:}

- Day 1: Vehicle and Powertrain Dynamics

- Day 2: Rail Vehicle Dynamics and Flexible Bodies

- Day 3: General MBS and R\&D Update

Sign up for individual days or the entire event join the sessions that interest you most.

Find out more:

events.3ds.com/MBS-Tech-Days
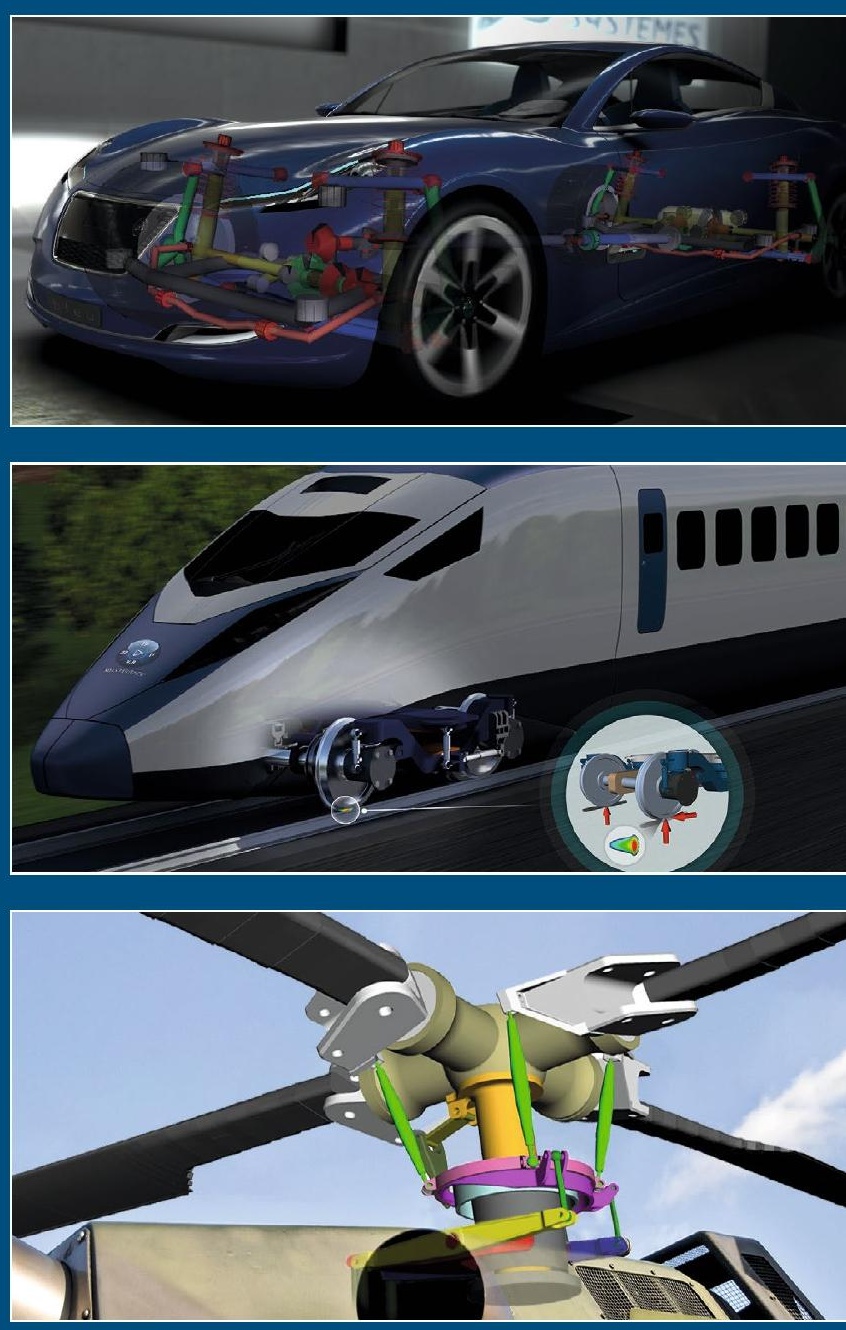
changes and adjustments in the traffic situation. The increasing number of electrically operated - and therefore very silent - automobiles requires other road users to pay even more visual attention already today. Additionally, the steadily growing number of electrically powered bicycles, which develop unusually high speeds, leads to new risks, especially in night-time road traffic.

The accident statistics of the past few years clearly reflect this: In 2020, the number of e-bike riders killed in traffic rose by $20 \%$, although all other driving accidents resulting in death fell due to the Covid-19 pandemic [5]. Since car drivers often tend to ignore the right of way with regard to more vulnerable road users, an earlier and clearer displaying of driving maneuvers using light projections can give the mentioned road users more time to react properly and help reducing the risk of accident, especially at night.

Additional mini projectors on cars or bicycles open up new technical possibilities for improving road safety. In the future, these projectors could be used to apply warnings directly onto the ground in the vicinity of the vehicle in order to provide information about turning or parking maneuvers, FIGURE 3.

\section{TECHNICAL SOLUTIONS FOR GROUND PROJECTIONS}

The type of desired projection will determine the technology to be used. Whether static, semi-dynamic or dynamic including videos will be just as decisive as the question of color: monochrome or color-

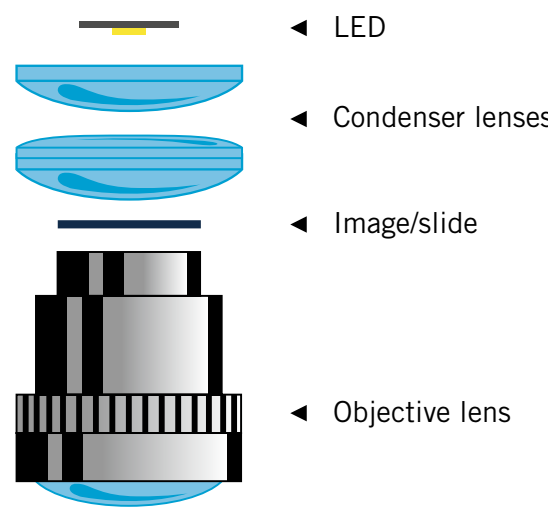

FIGURE 4 Principle of a static projector for simple slide or logo representation (C) Marelli Automotive Lighting | AS2)

ful? Not insignificant is furthermore the question of the representation: specified, selectable by the driver or to be uploaded individually? In this context, a personalization of the scenarios and the ability to update them are important issues. For the actual implementation, specifications such as size and position of the targeted illumination field, the luminance within the illumination field and the resolution of the representation will be added.

Static projections are already on the market - currently primarily for logo projection. The most common principle is that of the classic slide projector. In this case, a type of slide with the logo (image) to be displayed is illuminated by an LED and displayed with a simple lens, FIGURE 4. These logo projectors, which are a few centimeters in size, are available as original parts from the vehicle manufacturer or as retrofit kits in the aftermarket.

Other technical solutions for the implementation of static projections are based on micro lens arrays. Here, the resulting image is composed of individual partial depictions. This technology is therefore particularly suitable for projections at very flat angles. Both optical systems are built relatively simple, and their LEDs are supplied directly from the $12-\mathrm{V}$ vehicle electrical system or from batteries. Also semi-dynamic superimpositions of structures will therefore be possible through several static projectors connected in series. This means that projections improving traffic safety, such as an extended turn indicator, can be implemented in a technically simple and cost-effective manner.

If, however, multi-colored, dynamic projections are to be implemented on the roadway, HD projection systems based on DMD, LCD or micro-LED arrays, come into consideration. These systems are visually and electronically much more demanding, as they contain a digital image generator that needs to be illuminated and controlled accordingly,

FIGURE 5. In addition to displaying multi-colored videos, these systems offer the possibility of projecting various scenarios - also personalized and as Over-the-Air (OTA) updates.

The lighting requirements for fulfilling certain vehicle related functions are determined in coordination with the car manufacturers. It has not yet been decided which technology of mini projectors - considering various parameters - will win the race.
FIGURE 5 Principle of a dynamic projector for more complex multi-colored, dynamic video representation (C) Marelli Automotive Lighting | AS2)

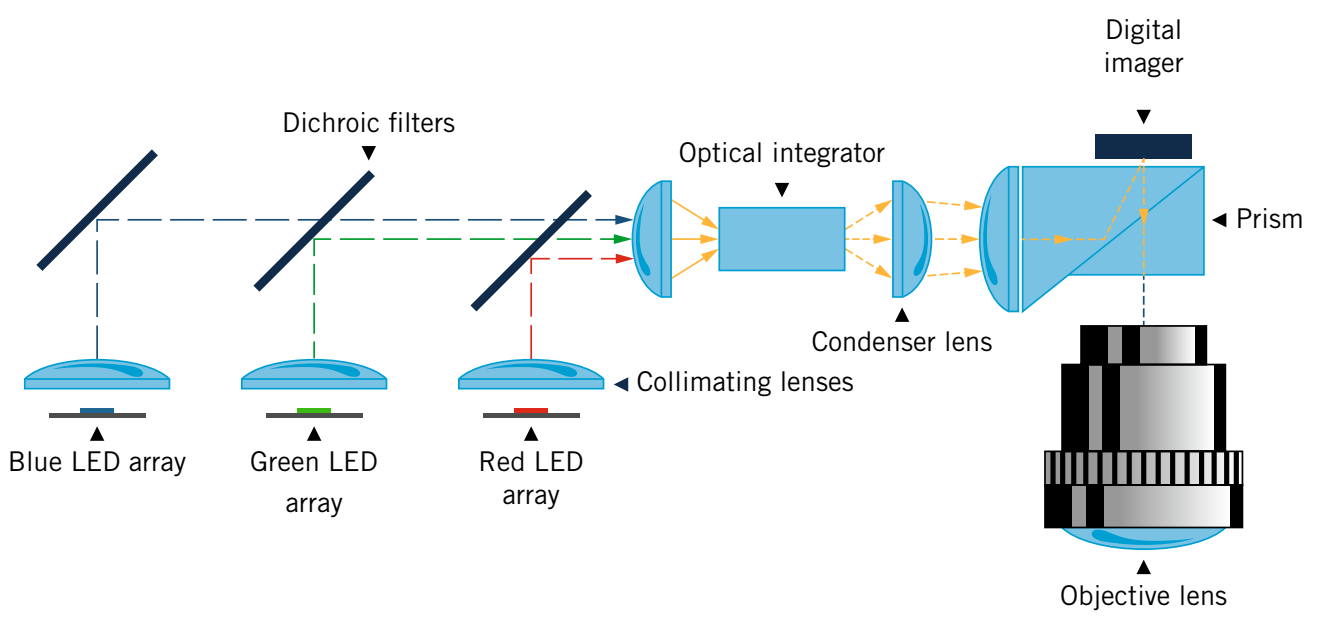




\section{INTEGRATION CONCEPTS OF THE GROUND PROJECTORS}

When integrating the light sources for ground projections into the vehicle, many other aspects must be observed. The setting up location must have enough installation space and be geometrically selected in such a way that the respective light cone can illuminate the desired projection area unhindered. A high emplacement location with regard to the road surface level such as for example the exterior mirror has the advantage that a large illumination field can be achieved with a relatively small cone of light. Due to the comparatively steep angle of incidence on the road, the trapezoidal correction in the illumination field is easy to control.

Other setting up locations such as doors, radiator grille, headlights or rear lights are also possible. In addition, there are various mechanical, electronic and thermal requirements, as well as resistance to dirt and splash water in order to avoid image disturbances.

\section{CONCLUSION}

Certainly, the more ambitious the light projections on the road, the more complex is the electronic control and the more important the connection to the vehicle architecture. It is up to the technical development to propose solutions for the future and to work on their implementation. The task of legislation, conversely, is to set the design framework accordingly so that the products are available for the market in good time.

\section{REFERENCES}

[1] Budanov, M.; Neumann, C.: Projektionen auf der Straße als Fahrerassistenz. Vortragsreihe Automobil-Beleuchtung, Haus der Technik, Berlin, 2018 [2] Hamm, M.: Glare Investigation and Safety Research on Digital Light Technologies. Lecture, SAE Congress, Detroit, 2019

[3] Khanh, T. Q.; Polin, D.: Investigation on Head lights with High-resolution Projection Modules. In: ATZworldwide 12/2018, pp. 70-72

[4] Rosenhahn, E.-O.; Link, F.: Traffic Safety Benefits provided by High Resolution Headlamp Systems. Lecture, ISAL, Darmstadt, 2019

[5] Deutsche Verkehrswacht: Deutlicher Anstieg be tödlichen Unfällen mit „E-Bikes“ - Verkehrswacht will mehr Aufklärung und Fahrtrainings. Press release, March 24, 2021
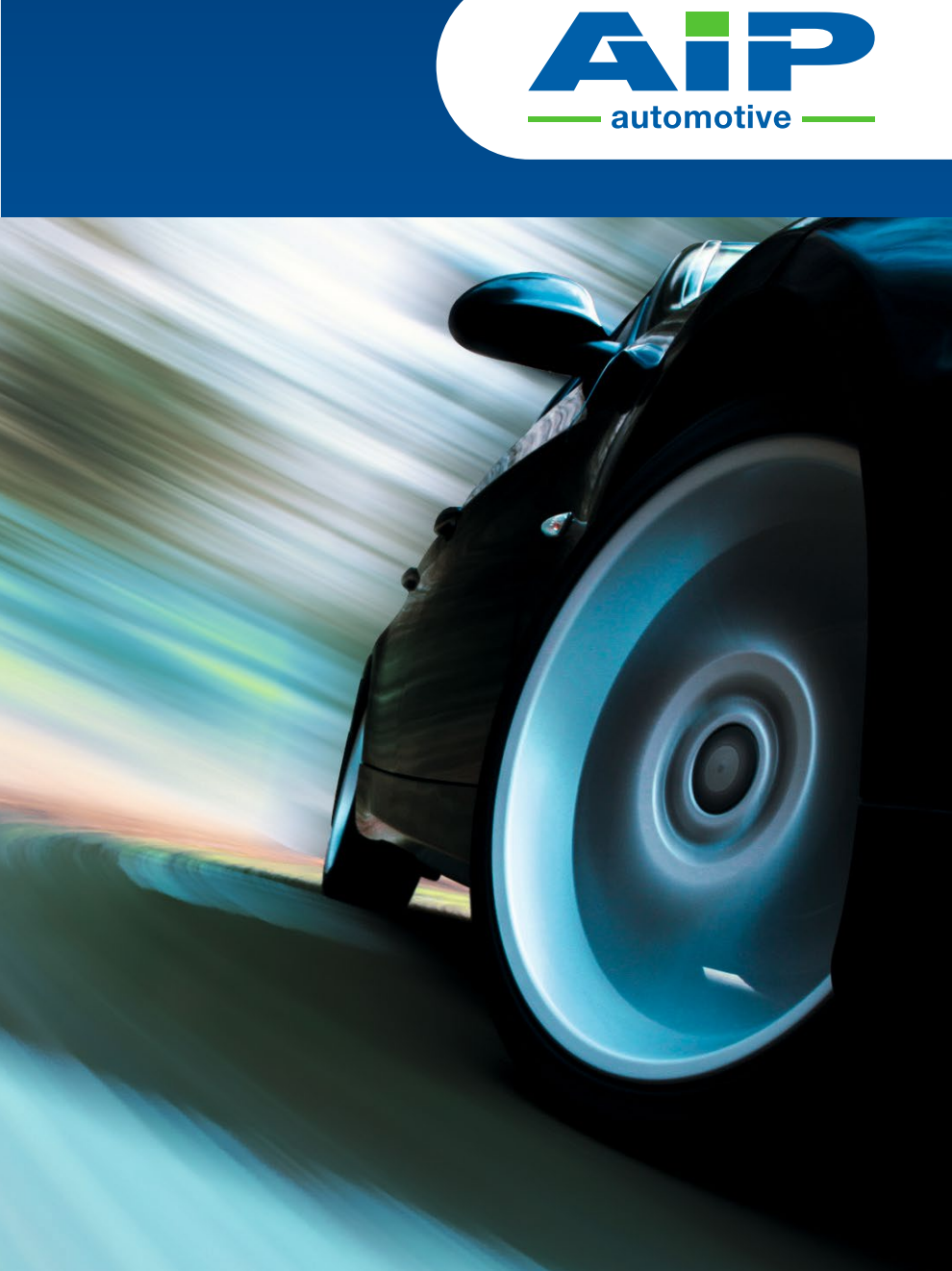

The Road in Your Lab

Pioneering Test Systems

for Road Driving Simulation

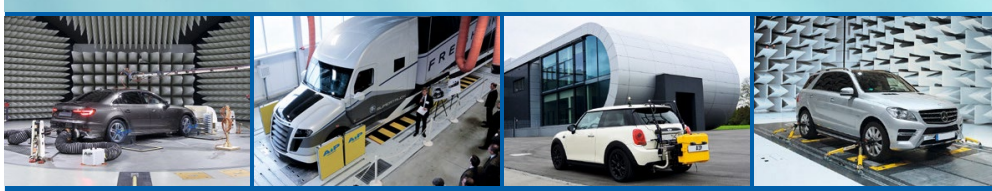

AIP designs and manufactures individual test systems for development and manufacturing centers, most vehicle OEM's and their tier suppliers as well as testing institutions, locally and globally.

AIP GmbH \& Co. KG

87490 Haldenwang / Germany

Tel. +49 (0)8374-2409-0

info@aip-automotive.de

www.aip-automotive.de

Test Systems for a Clean and Safe Environment 